\title{
ESTUDIO PALINOLÓGICO DE MIELES DE MONTAÑA DE LA PROVINCIA DE ORENSE (NW-ESPAÑA)
}

\author{
M. Isabel IGLESIAS, Ma Victoria JATO y M. Jesús AIRA
}

\begin{abstract}
RESUMEN. Estudio palinológico de mieles de montaña de la provincia de Orense (NO-España).Se analizan cualitativa y cuantitativamente trece muestras de miel procedentes de colmenares ubicados en la zona montañosa oriental de Galicia, entre los 600 y $1000 \mathrm{~m}$ de altitud. En once de las muestras, aparece como polen dominante Castanea sativa Miller, alcanzando valores en tres de ellas entre el $80-84 \%$. Rubus sp. es dominante en una de las mieles estudiadas, en la que alcanza un $67 \%$, mientras que las restantes muestras se consideran multiflorales, con abundancia, además de los táxones citados, de otros tipos polínicos como: Adenocarpus complicatus DC., Campanula sp., Hypericum perforatum L., Lotus corniculatus L., Prunus sp., Crataegus monogina Jacq. y Ericaceae.
\end{abstract}

Palabras clave. Galicia, miel, polen, caracterización.

ABSTRACT. Palynological study of mountain honey on the Orense province (NW Spain). In the following study a quantitative and qualitative analysis of thirteen samples of honey is realised. The honey is extracted from beehives all of which are located at an altitude between $600 \mathrm{~m}$ to $1000 \mathrm{~m}$ in the province of Orense (NW Spain). In eleven of the samples the dominant pollen is Castanea sativa, reaching in three of them a percentage between $80-84 \%$. The pollen of Rubus sp. is also dominant in one of honeys studied, wich a percentage of $67 \%$. The nine remaining honeys are polyfloral and as well as Castanea sativa and Rubus sp. other pollens are commonly present, these being: Adenocarpus complicatus, Campanula sp., Hypericum perforatum, Lotus corniculatus, Prunus sp., Crataegus monogina and Ericaceae.

Keys words. Galicia, honey, pollen, characterizacion.

\section{INTRODUCCIÓN}

Los primeros trabajos sobre mieles gallegas fueron llevados a cabo por Vieitez (1950), y en los últimos años se han aportado nuevos datos con los trabajos de Sánchez y Sáenz (1982); Terradillos (1988); Jato e Iglesias (1989); Aira, Ramil y Sáa Otero (1990); González Porto, Díaz Losada y Sáa Otero (1991); Sáa Otero, Ramil Rego y Aira
Rodríguez (1991); Jato, Salla-Llinares, Iglesias y Suárez-Cervera (1991).

En 1991, Aira et al., haciendo una revisión bibliográfica de los estudios llevados a cabo por diferentes autores, sobre un total de 215 mieles encuentran que un $50 \%$ de ellas son multiflorales y el otro $50 \%$ monoflorales, principalmente de Eucalyptus globulus Labill., Castanea sativa Miller y Rubus sp.

Mientras que las mieles de Eucalyptus se 


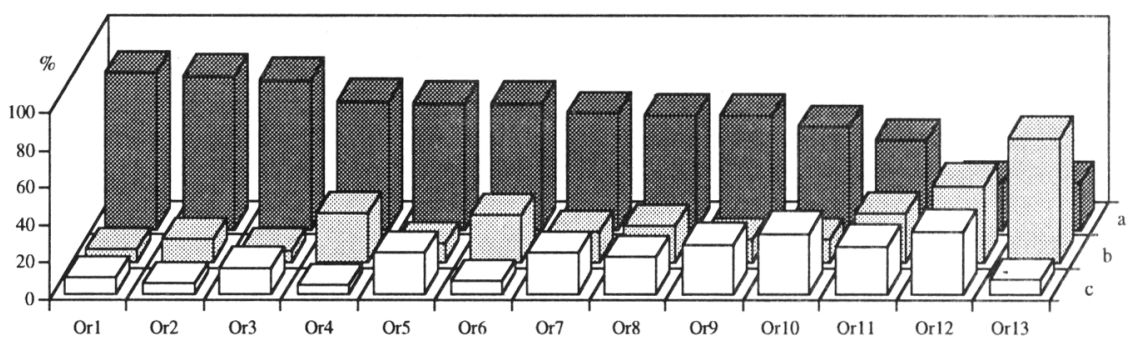

Figura 1. Porcentajes alcanzados por Castanea sativa (a), Rubus sp. (b) y otros táxones identificados (c), en las diferentes muestras estudiadas.

producen en las zonas costeras, ligadas a la presencia de amplias extensiones repobladas por este taxon, las de Castanea y Rubus son mieles cosechadas en el interior de Galicia. Finalmente las de brezo aparecen ligadas a zonas de montaña.

\section{MATERIAL Y MÉTODOS}

Se han analizado trece muestras de miel, procedentes de otros tantos colmenares ubicados en la provincia de Orense, en zonas comprendidas entre los 600 y 1000 metros de altitud. Todas ellas pertenecen a la cosecha de 1989 y el método de extración utilizado ha sido por centrifugación.

La metodología empleada es la recomendada por la Comisión Internacional de Botánica Apícola descrita por Louveaux, Maurizio \& Vorwohl (1978), utilizando el método de extración sin acetolisis y tiñendo con fuchina básica.

Se ha realizado el análisis cualitativo y cuantitativo y, atendiendo a los criterios de Vergeron (1964), se han contado 1200 granos de polen por muestra.

Como material de referencia se utilizaron preparaciones microscópicas de la flora más abundante en los alrededores de los colmenares.

Todas las preparaciones microscópicas de las mieles analizadas, se encuentran archi- vadas en la Palinoteca de la Escuela Universitaria de E.G.B. de Orense, Departamento de Recursos Naturales y Medio Ambiente de la Universidad de Vigo.

\section{RESULTADOS}

Los resultados obtenidos aparecen reflejados en la tabla 1, en la que figuran, para cada miel, los tipos polínicos y sus frecuencias, número de granos de polen contabilizados para el análisis cualitativo, número de tipos polínicos identificados, clase de Maurizio a la que pertenecen y procedimiento de extración de la miel.

En la figura 1, se representan los porcentajes alcanzados por Rubus sp. y Castanea sativa, los dos táxones de mayor importancia en las mieles estudiadas, en relación a los porcentajes de los demás táxones identificados.

\section{Análisis cualitativo}

El número máximo de tipos polínicos identificados es de 38 y el mínimo de 19 obteniéndose como media 28 formas por muestra. Aplicando el criterio de Pérez de Zabalza (1989), se corresponden con una riqueza polínica alta.

Los tipos polínicos presentes en todas las 


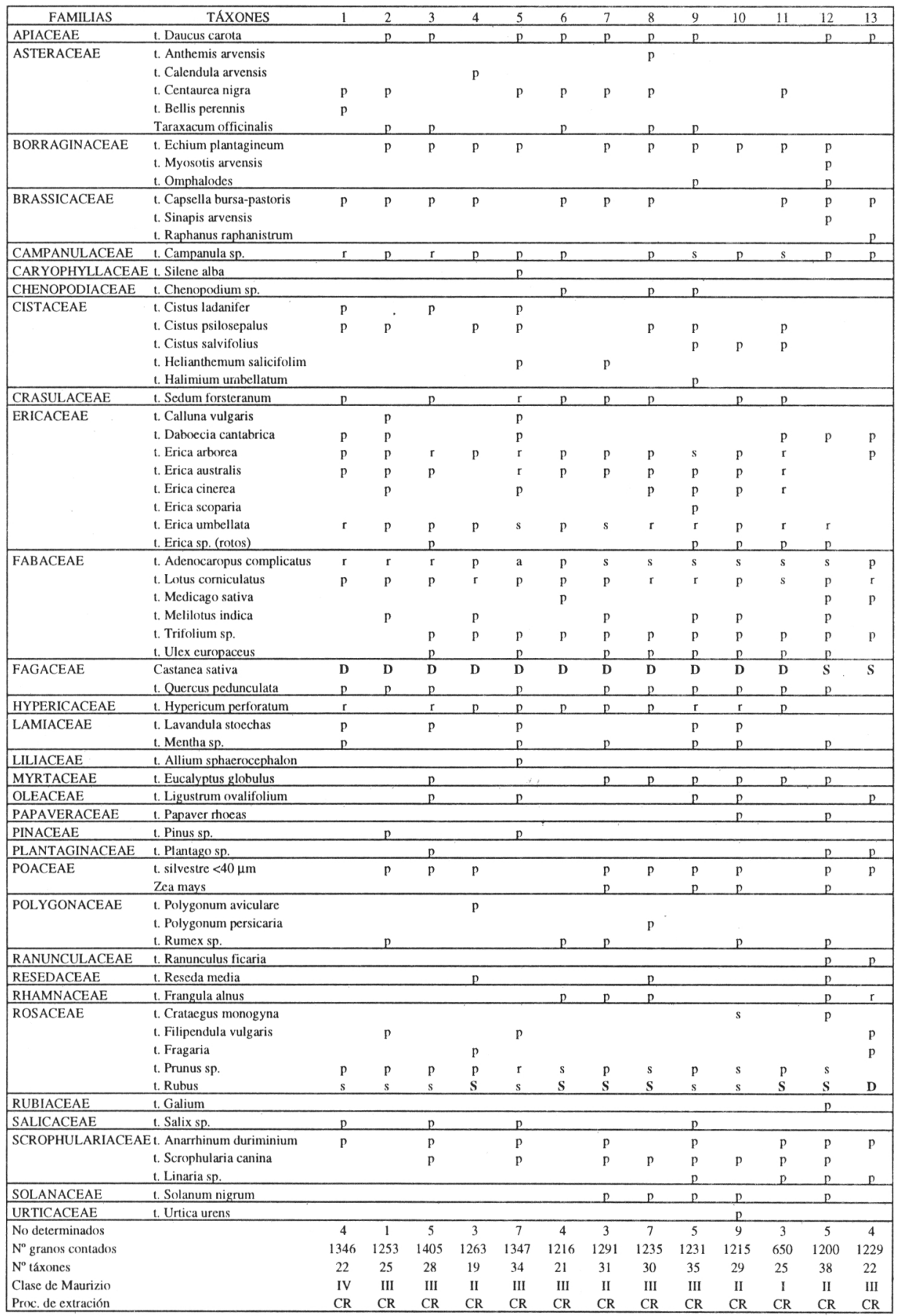

Tabla 1. Espectros polínicos de las mieles analizadas. D: Polen dominante $(>45 \%)$; S: Secundario (16$45 \%)$; s: De menor importancia (3-15\%); r: Raro (1-3\%); p: Presente. De 1 a 13, muestras de mieles (Or). 
muestras estudiadas, además de Castanea sativa y Rubus sp., son: Adenocarpus complicatus.DC, Lotus corniculatus L., Erica umbellata L., Erica arborea L. y Prunus sp., presentes en 12 de ellas. Otros tipos polínicos identificados en el $61 \%$ de la muestras son: Centaurea nigra L., Echium plantagineum L., Capsella bursa-pastoris L., Erica australis L., Trifolium sp., Ulex europaeus L., Quercus pedunculata L., Hypericum perforatum, Anarrhinum duriminium Desf., Scrophularia canina L. y Poaceae.

\section{Análisis cuantitativo}

De acuerdo con las clases propuestas por Maurizio (1939), el 58,8\% de las muestras corresponden a la clase III (10.000/50.000 granos de polen por gramo de miel), el $30,7 \%$ a la clase II (2.000/10.000), el 7,6\% a la clase I y en igual porcentaje para la clase IV.

\section{DISCUSIÓN}

Miel de castaño.- Tres de las muestras analizadas (las referenciadas como Or-1, Or2 , Or-3) presentan valores superiores al $80 \%$ de Castanea sativa, por ello han sido consideradas como monoflorales de castaño (Maurizio \& Louveaux, 1965; Gómez Ferreras, 1985; Pérez de Zabalza y Gómez Ferreras, 1988). Acompañando a Castanea sativa y como polen de menor importancia Rubus sp. (3-15\%), Adenocarpus complicatus como polen raro (1$3 \%)$, Erica umbellata y Prunus sp. como raro (1-3\%) o presente $(<1 \%)$.

Miel de Rubus.- La muestra referenciada como (Or-13) presenta como tipo dominante Rubus sp., con un 67\%, acompañado de Castanea sativa (25\%), Lotus corniculatus y Frangula alnus Miller como polen raro y esporádico respectivamente.

Mieles multiflorales.- Las nueve muestras restantes han sido así tipificadas, en ellas destacamos Castanea sativa con porcentajes comprendidos entre 55 y $69 \%$, acompañado de Rubus sp. (10 al 41\%), junto con Lotus corniculatus, Campanula sp., Adenocarpus complicatus, Erica umbellata y Prunus sp. como pólenes de menor importancia, raro o presente, según las mieles, y Trifolium sp. siempre como polen presente.

\section{CONCLUSIONES}

La presencia constante de Castanea sativa y Rubus sp. ponen en evidencia la importancia que ambos táxones tienen en la provincia orensana. La gran atracción que siente la abeja por el polen de castaño y la época en que tiene lugar su floración (entre la segunda quincena del mes de Junio y la primera de Julio) explican su elevada presencia.

Por otro lado, aunque no existen en la actualidad grandes superficies de Castanea sativa, éstas ocupan principalmente zonas de clima subcontinental en donde pueden encontrarse en estado climácico, especialmente en pendientes escarpadas de distintas zonas de la provincia de Orense.

Por lo que respecta a Rubus sp., es un taxon frecuente en toda nuestra geografía, en donde forma parte del matorral de bosque y muy frecuente en bordes de caminos, prados etc. Su período de floración se extiende durante todo el verano y coincide con un momento de escasez de otras fuentes nectaríferas de mayor apetencia para la abeja, por lo que en ocasiones se puede considerar como la única fuente disponible en dicho período.

Por otra parte se confirma de nuevo la combinación Castanea-Rubus-Lotus-Adenocarpus-Prunus-Campanula, citada por Jato e Iglesias (1990) y Jato, Sala-Llinares, Iglesias y Suárez-Cervera (1991) para mieles de la provincia de Orense, presente en el $92 \%$ de las mieles que se incluyen en este trabajo. 


\section{BIBLIOGRAFÍA}

AIRA RODRIGUEZ, M.J., P. RAMIL REGO y M.P. SÁA OTERO -1990- Identificación polínica de Ericaceae en mieles gallegas. Acta Botánica Malacitana, 15: 27-32.

GÓMEZ FERRERAS, C. -1985-Estudio polínico de mieles españolas. Tesis Doctoral. Universidad Complutense. Madrid.

GONZÁLEZ PORTO, A.V., E. DÍAZ LOSADA y M.P. SÁA OTERO -1991- Espectro polínico de mieles orensanas. Vida Apícola, 49: 47-51.

JATO, M.V. e I. IGLESIAS -1989- Oscilaciones del contenido polínico de mieles orensanas. Apiacta, XXIV: 82-86

JATO, M.V., A. SALLA-LLINARES, M.I. IGLESIAS y M. SUÁREZ-CERVERA -1991Pollens of the honeys fron norhwestern Spain. Journal of Apicultural Research. 30(2): 69-73.

LOUVEAUX, J.A., A. MAURIZIO \& G. VORWOHL -1978 - Methods of melyssopalynology. Bee World, 59(4): 139157.

MAURIZIO, A.-1939- Untersuchengen zur quantitativen pollen analyse des honigs. Mitt. Geb. Lebensmitelters, 30(1-3): 27-69.

MAURIZIO, A \& J. LOUVEAUX - 1965- Pollens de plantes melliféres d'Europe. Unión des groupements Apicoles Français, Paris 148 pp.

PÉREZ DE ZABALZA, A.I. y C. GÓMEZ FERRERAS -1988- Análisis polínico de mieles de la Navarra húmeda del noroeste. Acta Salmanticiensa Bib. Ciencias, 65: 239-245.
PÉREZ DE ZABALZA, A.I. -1989- Estudio palinológico de las mieles de Navarra. Tesis Doctoral. Universidad de Navarra. 385 pp.

SÁA OTERO, M.P., P. RAMIL REGO y M.J. AIRA RODRIGUEZ - 1991- Análisis polínico de mieles procedentes de las provincias de Lugo y Orense. Nova Acta Científica Compostelana (Biología). 2: 57-63.

SÁNCHEZCUNQUEIRO, C. y C. SÁENZLAINZ -1982- Análisis polínico de mieles de Pontevedra. Lazaroa, 4: 253-268.

TERRADILLOS, L.A. -1988- Estudio microscópico del sedimento de la miel de galicia. Tesis Doctoral. Universidad de Santiago. 232 pp.

VERGERON, P. -1964- Interpretation statistique des résultats en matière d'analyse pollinique des miels. Ann. Abeille, 7(4): 349-364.

VIEITEZ, E. -1950- Palynological observations on some Spanish Honeys. Bul. Torrey Bot. Club, 77(6): 495-502.

Aceptado para su publicación en Junio de 1993

Dirección de los autores. M.I. Iglesias y M.V. Jato: Dpto. de Recursos Naturales y Medio Ambiente. Universidad de Vigo. M.J. Aira: Dpto. de Biología Vegetal. Universidad de Santiago. 15706 Santiago. 